\title{
Automatic Pose Tracking and Motion Transfer to Arbitrary 3D Characters
}

\author{
Ju Shen ${ }^{1(\bowtie)}$ and Jianjun Yang ${ }^{2}$ \\ 1 Department of Computer Science, University of Dayton, Dayton, OH 45469, USA \\ jshen1@udayton. edu \\ 2 Department of Computer Science and Information Systems, \\ University of North Georgia, Oakwood, GA 30566, USA
}

\begin{abstract}
D character with human motion offers a high end technology for creating compelling contents in graphics. In this paper, we present an automatic system to animate a $3 \mathrm{D}$ character with human motion streamed from a single video camera. The traditional mesh animation process is laborious and requires high skills from the users. To mitigate this limitation, a new way for bringing $3 \mathrm{D}$ objects to life is introduced that does not need explicit mesh positioning. In our framework, the animation is driven by the captured motion from an ordinary RGB camera. In order to reduce the ambiguity of the estimated 3D pose, a modified spatio-temporal constraint based algorithm is used for articulated gesture estimation across frames while maintaining temporal coherence. Our approach demonstrates promising performance on par with state-of-theart techniques. We believe the presented animation system will allow a new audience of novice users to easily and efficiently create animation for arbitrary $3 \mathrm{D}$ characters.
\end{abstract}

Keywords: Monocular pose estimation $\cdot 3 \mathrm{D}$ animation $\cdot$ Motion retargeting $\cdot$ Motion transfer

\section{Introduction}

Human-like animation for arbitrary 3D characters provides a high end technology in film and game production. State-of-the-art techniques demonstrate impressive animation results $[1,2]$. However, most of the methods require tedious manual work and professional skills from experienced users, which greatly hinders its practical adoption in consumer level applications. The latest user-interventionfree techniques often rely on strong assumptions and additional constraints about how the character is presented by compromising its potential flexibility and robustness. The disadvantage of these these approaches are further addressed in [23]. With the wide spread of commodity cameras, such as a webcam from mobile devices, performance-driven animation become a popular technique. By mapping the detected human pose to the character's skeletons, it allows novice users to effectively generate expressive character motions with their own body movements (Fig. 1).

(C) Springer International Publishing Switzerland 2015

Y.-J. Zhang (Ed.): ICIG 2015, Part I, LNCS 9217, pp. 640-653, 2015.

DOI: $10.1007 / 978-3-319-21978-3 \_56$ 

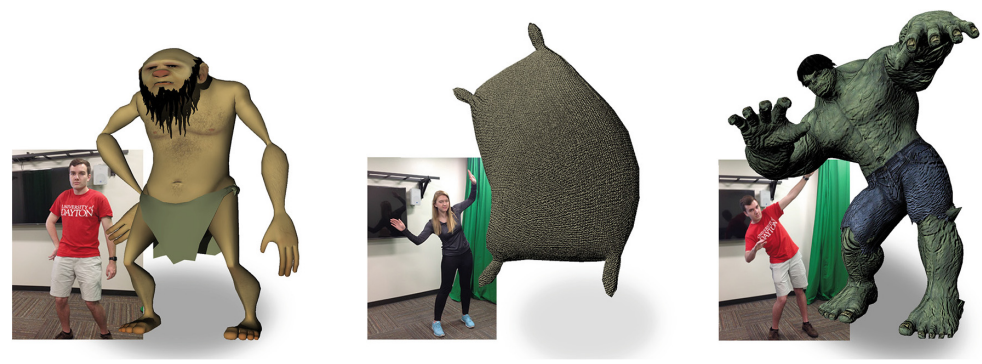

Fig. 1. Motion transfer from video to 3D characters

Instead of the traditional input from keyboard or mouse, this new source makes the animation process more intuitive and efficient by speeding up the crafted animation process. Another advantage of such a performance driven system is to ensure more realistic body motions with temporal consistent human body movement. While many different techniques exist for body tracking and motion transfer, most of the methods reply on the tracked markers. They typically assume that the body shape of the source data closely resembles that of the target animated character, which is not often true for many scenarios. The recent advent of RGB-D cameras, such as Microsoft Kinect, has enabled a number of non-intrusive body tracking methods $[3,4,16]$. This algorithm achieves realtime performance and shows robust and accurate results than previous methods. However, both the markers and depth sensors based methods require additional resources that limit their practical applications. In contrast, regular RGB cameras would favor more ordinary users as they are wildly available and easy-to-use.

On the other hand, it is not trivial to achieve fully automatic human-like 3D animation from regular RGB cameras: first, the automatic recovery of 3D human pose from a single, regular video cameras is a very challenging problem due to the potential ambiguities from monocular vision. The occlusion or selfocclusion, body deformation, light variations, diverse poses, and highly dynamic environments could lead to inaccurate estimation on human body articulations. To resolve this problem, there are generally two types of methods: data-driven and structure from motion based techniques. For data-driven methods, the 3D poses are usually estimated from the detected $2 \mathrm{D}$ joints. For the structure from motion methods, the 3D positions are computed by finding correspondences across frames. In this paper, we integrate both these strategies. Another challenge for RGB camera based performance driven problem is the mapping between the extracted human poses to a 3D character. Especially when the target character is non-humanoid, direct human skeletal control would be difficult. So the motion re-targeting solution should be generic and account for all possible structurally dissimilar characters.

In this paper, we envision a system for automatic motion tracking from an RGB camera and generating plausible human-like animation for arbitrary 3D characters. First, human motion is captured directly by a monocular video (e.g. a web camera). 3D poses are estimated from the captured data, which are used for 
the motion transfer to a target character. Meanwhile, as a parallel process, for an input 3D character, we decompose the unknown mesh into multiple components through a sparse graph construction, followed by a tree structure specification to extract the potential embedded skeletons. To achieve an intuitive mapping in the structurally different motion spaces, a flexible and user friendly interface is provided to interactively define a few (less than 6) pose correspondences between the source and target objects. We believe our animation system captures people's imagination and provides a wide range of scenarios: imagine in the video games, players can control 3D avatars with their own bodies, without any manual rigging or skinning process, as well as leveraging these types of playful mesh animations for physics-enabled gaming; other scenarios include designers can animate characters by simply acting in front an ordinary web camera. We conclude by discussing qualitative experiences of using the system, its strengths and limitations, and directions for future work.

The rest of this paper is structured as follows. Section 2 gives a brief overview of the literatures on character animation and skeleton extraction. In Sect. 3, we give an overview of our system, including skeleton extraction from 3D mesh, automatic animation, and pose estimation from monocular. The technical details are presented in the following Sects. 5 and 6. Section 6 demonstrates experiment results and Sect. 7 concludes the paper with some discussion of future work.

\section{Related Work}

The literature contains a plethora of studies on 3D character rigging and animation. A typical animation technique is to extract the potential skeleton from a given 3D mesh according to the geometry. Animation is generated by modifying the embeded skeletons to drive deformation of the attached skins. Bloomenthal et al. propose a technique by converting the geometric structure to IK skeletons to facilitate the animation process [5]. However, the IK skeleton often need nonlinear constraints to articulately estimate the potential poses. Skeletons can also be inferred by using graph-based structure. The nodes are determined according to the mesh surface [6], or from the amount of volumes [7]. Katz et al. extract a skeleton from a meshed model using a hierarchical decomposition of this mesh into meaningful parts. However the proposed model assumes skeletons are in star-shape, which is not always true and not applicable for realistic animation. Lien et al. propose a shape decomposition method for skeleton extraction [8]. The skeleton is computed based on the centroids and principal axes of the shape's components, which produce reasonable results. Our work share a similar decomposition based strategy to infer the embedded skeletons. However, instead of computing the centroids, we use the vertex connectivity and geometric distribution as a cue to divide the mesh into multiple components.

For the motion source, color image is a possible and common input media, from which body poses can be identified automatically. There have been extensive studies about image recognition, such as the segmentation based methods [9$11]$, or feature based methods [12,13], or learning based method [14,15, 19]. However, for a single image, due to the color variations, occlusion, or self-occlusion 
issues, it is difficult to estimate the pose articulately and accurately. The recent advent of RGB-D cameras regain the interests of performance driven for animation. Using active depth sensors for mocap becomes a popular topic in the last few years. In particular Microsoft Kinect contributes a growing interest in realtime depth acquisition and has enabled many human-computer interactive applications [16]. Despite its advantages of RGB-D camera systems, the hardware is not widely accessible for ordinary users. On the other hand, regular video can be easily obtained nowadays from webcam to mobile devices. However it is difficult to achieve accurate pose tracking and animation from a single RGB camera. The typical method is to reply on $2 \mathrm{D}$ correspondences through a set of images / frames via applying a factorization method, which is firstly introduced in for reconstructing the 3D pose of a rigid structure. Bregler et al. introduce a factorization method for non-rigid structures [17]. As a further improvement, Yang et al. propose a pictorial structures based flexible mixtures-of-parts to estimate articulated pose from color images [18]. However this approach fails in the scenario of self-occlusion. In this paper, we introduce a simple but effective solution to reduce the ambiguity by simultaneously processing multiple frames from a captured footage.

\section{Approach Overview}

In this section, we briefly describe the workflow of the animation process in our system. As shown in Fig. 2, there are two input: a sequence of video frames and a given unknown 3D character. The video frames can be obtained from a web camera without any calibration procedure required. For the input 3D character, it consists of a collection of triangles or polygons. From the input video footage, the actor's 3D gestures are detected by the pose estimation box. The extracted pose sequences are used to drive the $3 \mathrm{D}$ character to animate.

From the workflow diagram, one can see there are four stages involved: pose estimation, skeleton extraction, skin attachment, and pose skeleton mapping. Our main work concentrates on the first two stages: pose estimation and skeleton extraction. To accurate detect the poses from a video, we use a modified spatio-temporal parsing technique by processing multiple frames simultaneously to remove the estimated pose ambiguity. As a parallel procedure, the potential skeleton of the input character is extracted according to the 3D mesh. We use a sparse graph to model the skeleton joint distribution, by which all the 3D vertices can be decomposed into multiple components. The output graph is further refined to a desirable skeleton structure by merging possible nodes in the graph. In the skin attachment stage, weight assignment for each vertex is carried out based on the heat equilibrium [33]. During the animation, linear blend skinning (LBS) algorithm is used to transform the vertices to new positions. For the correspondence mapping, our system allows user to select the joint correspondences between the detected poses from the original video and the extracted skeletons from the target character. A key frame is selected to get a starting 


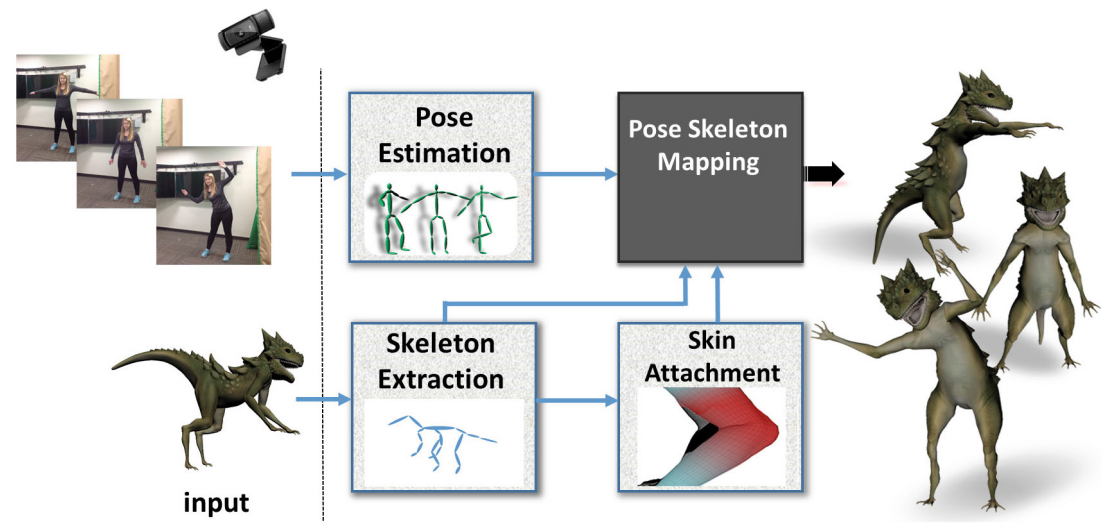

Fig. 2. The workflow of our approach

pose for the subject. This pose is used to map the initial status of the target character. During video tracking, the angle change of each joint is used as a cue to transform the target skeleton accordingly.

In the following sections, we describe the technical details about pose estimation and skeleton extraction.

\section{Pose Estimation from Monocular}

\subsection{Pictorial Structures}

To achieve fully automatic 2D human pose tracking from a video, we start with the state-of-the-art mixture of parts detectors by representing the human body parts as pictorial structures [27]. Based on the algorithm, for a single RGB image, the score of a specific pose configuration is defined as:

$$
S(I, l, t)=S(t)+\sum_{i \in V^{(I)}} \omega_{i}^{t_{i}} \cdot \phi\left(I, l_{i}\right)+\sum_{i, j \in E^{(I)}} \omega_{i, j}^{t_{i}, t_{j}} \cdot \psi\left(l_{i}, l_{j}\right)
$$

where $\phi\left(I, l_{i}\right)$ is the HOG descriptor extracted from location $l_{i}$ in the image $I$. The first term represents the regression score measuring the certainty a body part $i$ locates at $l_{i}$ based on texture mapping; the second term is the spatial score that the two adjacent parts $i$ and $j$ are from locations $l_{i}$ and $l_{j}$, which encode standard physical spring model. By maximizing the score over the locations of body parts $l_{i}$ for types $t$, an optimized pose configuration can be obtained for the image. This technique works well for a single image, which is used as the initial pose estimation in our system.

\subsection{Multiple Frames Processing}

After obtaining the initial pose from the video, the appearance for each body part can be learned by using the image parsing model [29]. For a part $l_{i}$, the 
sparse image $I_{S(i)}$ is produced by convolving its regression probability with the oriented image patches $s_{i}$. So according to the sparse image, for a given pixel color $c$, it delivers a probability that $c$ is from a particular part $i$, denoted as $P\left(l_{i} \mid c\right)$. The motivation of learning the color model is to incorporate appearance consistency across multiple frames [28]. Since the same person is captured in the video footage, the appearance of the body should remain similar among all the frames. We conduct the pose inference across multiple frames instead of treating each individual frame independently. In this way, it ensures smooth body movement in the temporal space. Furthermore, this batch processing scheme can offer effective ambiguity reduction on the articulated body parts.

Here we apply the spatio-temporal parsing technique by extending a singleframe pose estimation model to processing multiple frames simultaneously [30]. The basic idea is all the frames in the video contributes the pose estimation, i.g. both the subsequent and previous frames have influence on the current frame inference. The goal is to output a posterior for each frame $t$ with the probability $P\left(l_{i}^{t} \mid I^{t}\right)$ for every possible location $(x, y)$ associated to a body part $i$. Given all the frames $\left\{I_{t}\right\}$, our modified posterior metric for the body configurations over all the frames can be expressed as:

$$
\begin{array}{r}
P\left(\left\{l^{t}\right\} \mid\left\{I^{t}\right\}\right) \propto \exp \left(\sum _ { t , i } \left(\sum_{j \mid(i, j) \in E} \Psi\left(l_{i}^{t}, l_{j}^{t}\right)+\Phi\left(l_{i}^{t}\right)+\Omega\left(l_{i}^{t}, l_{i}^{t+1}\right)\right.\right. \\
\left.\left.+\Lambda\left(l_{\text {lua }}^{t}, l_{\text {rua }}^{t}\right)+\Lambda\left(l_{\text {lla }}^{t}, l_{\text {rla }}^{t}\right)+\Lambda\left(l_{\text {lul }}^{t}, l_{\text {rul }}^{t}\right)+\Lambda\left(l_{\text {lll }}^{t}, l_{\text {rll }}^{t}\right)\right)\right)
\end{array}
$$

where the spatial prior $\Psi(\cdot, \cdot)$ specifies the pairwise constrains on two connecting parts $l_{i}$ and $l_{j}$ by incorporating the kinematic properties. The likelihood $\Phi(\cdot)$ corresponds to the local image evidence for a part $l_{i}$ locates on a particular
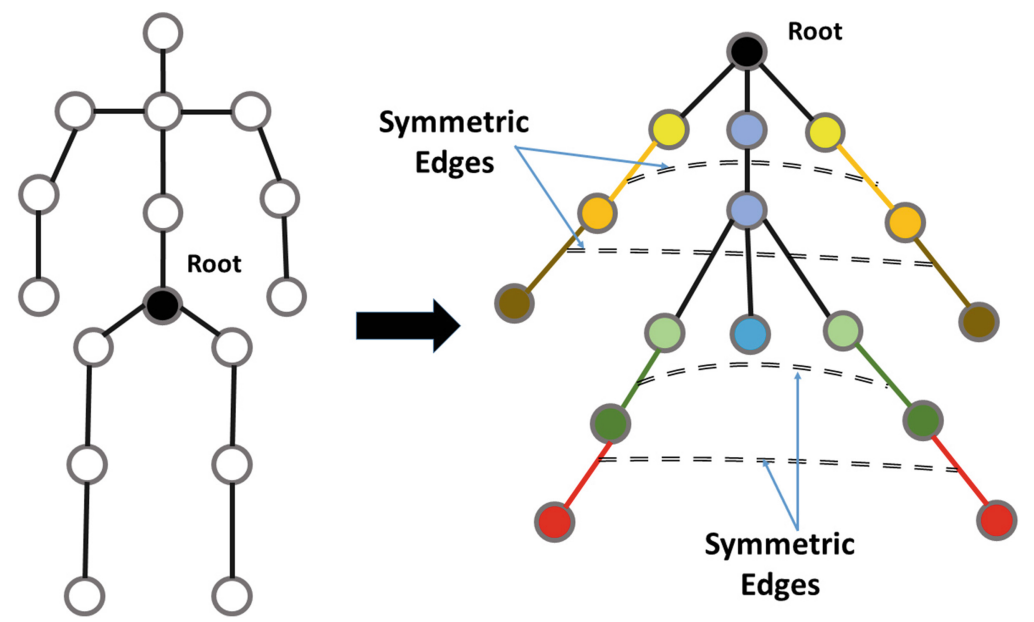

Fig. 3. Symmetric constraints by adding four additional edges across left and right limbs 
position of frame $t$. The temporal prior $\Omega$ encodes the temporal dependency between consecutive frames $t$ and $t+1$. Here, we use the same box-shaped distribution to encourage temporal coherence, the details of which are explained in [30]. The last four terms $\Lambda\left(l_{i}^{t}, l_{j}^{t}\right)$, where $i, j \in\{l u a, \ldots, r l l\}$ defines the Repulsive Edges, The motivation of using repulsive edges is to alleviate the double-counting problem occurs in the traditional kinematic model [31]. By adding four edges to the upper and lower limbs in the original kinematic tree (see Fig. 3), the new kinematic graph encodes the symmetric feature of human body that the left upper arm (lua) and left lower arm (lla) has similar appearances as the right upper arm (rua) and the right lower arm (rla). The same rule applies to the left/right legs $(l u l, l l l, r u l, r l l)$. Based on the symmetric tendency, we enforces the repulsive prior $\Lambda\left(l_{i}^{t}, l_{j}^{t}\right)$ to favor the body configuration with similar appearance between the left and right limbs and discourage the two side limbs overlap each other. This configuration preference can be mathematically interpreted in terms of penalties:

$$
\Lambda\left(l_{i}^{t}, l_{j}^{t}\right)= \begin{cases}\omega_{C}, & \text { if }\left\|I_{S(i)}^{t}-I_{S(j)}^{t}\right\| \geq \tau_{C} \\ \omega_{O}, & \text { if }\left\|l_{i}^{t}-l_{j}^{t}\right\| \leq \tau_{O} \\ 0, & \text { otherwise }\end{cases}
$$

where $\omega_{C}$ and $\omega_{O}$ represent the color consistency and location overlapping penalties. The related thresholds $\tau_{C}$ and $\tau_{O}$ are experimentally determined and can be manually adjusted depending on how strong does the system favor the consistency and parts separation.

To solve the above equations, the optimal configuration for the graph model can be approximated by using Loopy Belief Propagation. Experiments turned out this modified version of joint spatio-temporal inference, can disambiguate the estimated articulations that outperforms pose inference on individual frames.

\subsection{Re-Projection from $2 \mathrm{D}$ to $3 \mathrm{D}$}

To estimate the $3 \mathrm{D}$ pose, we start with the $2 \mathrm{D}$ joints output from the multiple frame processing procedure. The $3 \mathrm{D}$ pose is parameterized as a vector $\hat{\boldsymbol{L}}=$ $\left[\hat{l}_{1}^{T}, \ldots, \hat{l}_{n}^{T}\right]$ of $n 3 \mathrm{D}$ points corresponding to the $2 \mathrm{D}$ joints $\boldsymbol{L}=\left[l_{1}^{T}, \ldots, l_{n}^{T}\right]$. The 3D pose retrieval can be considered as a solution of a linear system, if multiple input images are available. We assume the camera's intrinsic parameters $\boldsymbol{K}$ to be unknown. The projection of a $3 \mathrm{D}$ point $\hat{l}_{i}$ onto its $2 \mathrm{D}$ position $l_{i}$ can be written as a function of the unknown parameters $\boldsymbol{K}$ and $\hat{l}_{i}$ :

$$
s_{i}\left[l_{i}^{T} 1\right]^{T}=\boldsymbol{K} \hat{l}_{i}
$$

where $s_{i}$ is a projection scalar. With $n$ estimated joints location for each frame, we obtain $n$ such linear equations. As we do the joint inference on the whole video sequence rather than a single frame, correspondences can be established across different frames. These correspondences together with the kinematic constraints are used jointly to solve the linear equations. We refer reader the N-points parameter determination for further details [32]. 


\section{Skeleton Extraction from 3D Mesh}

As a parallel procedure, our system aims to extract the potential skeleton structure from an unknown 3D character. To achieve this goal, we first decompose the input 3D mesh into multiple components according to the polygons' connectivity and geometric distributions. The decomposed components can be represented as a graph, denoted as $\mathcal{G}(V, E)$. Every node $v_{i} \in V$ corresponds to a component. For any two neighboring nodes $v_{i}$ and $v_{j}$, there is an edge $e_{i j} \in E$ connecting them.

So each polygon is assigned to a certain component or labeled by a node $v_{i}$ in the graph. This polygon labeling task is accomplished through an iterative procedure [20]. Starting from any polygon, $\alpha$-expansion is performed by minimizing the discontinuity-preserving energy. All the traversed polygons during the region expansion are assigned to the same component. Repeat the same process for the remaining polygons until the whole mesh are decomposed into multiple components $V$. For any two nodes $v_{i}, v_{j} \in V$, an edge $e_{i j}$ is drawn if there is no oriented bounding box collision between $v_{i}$ and $v_{j}$. For the graph construction, readers can also refer other techniques [34].

Sparse Graph Generation. The generated graph representation $\mathcal{G}(V, E)$, can be considered as a coarse skeletal structure for the input mesh. However, the resolution of the graph and its connectivity can be potentially complex and over detailed with redundant nodes and edges. A direct use of such a graph as the skeleton could yield unsatisfactory results [21]. To further simplify the structure, a node merging procedure is carried out based on the Quadratic Error Metric (QEM), which is often used for surface simplification [22]. Let a $3 \times 1$ vector $\boldsymbol{c}_{i}=\left[x_{i}, y_{i}, z_{i}\right]^{T}$ be the mass center of the point cloud of the node $v_{i}$. If any two nodes $v_{i}$ and $v_{j}$ can be merged into a new node $\hat{v}$, we require their corresponding edge $e_{i j}$ must satisfy the following criteria:

$$
\operatorname{merge}\left(v_{i}, v_{j}\right) \Rightarrow\left\{\begin{array}{l}
\operatorname{deg}\left(v_{i}\right)>1 \cap \operatorname{deg}\left(v_{j}\right)>1 \\
\exists\left\{p_{t}\right\}, p_{t} \subset\{V\} \text { s.t. } v_{i} \stackrel{p_{t}}{\longrightarrow} v_{j} \cap\left|\left\{p_{t}\right\}\right|>1
\end{array}\right.
$$

where $p_{t}$ represents a path connecting nodes $v_{i}$ and $v_{j}$. The operator $|\cdot|$ returns the total number of an input set. This node merging criterion encodes the spatial constraint that discourage the skeleton to have over-dense components by limiting the node degree (criterion 1) and the distance to its neighbors (criterion 2). Based on the work [20], the metrics below are to determine whether two nodes $v_{i}$ and $v_{j}$ are qualified for merging.

To identify the potential merging pair of nodes $v_{i}$ and $v_{j}$, we iteratively assign a cost value for each edge $e_{i j}$ on the graph. The edge cost term $\mathcal{C}$ is defined as

$$
\mathcal{C}\left(e_{i j}\right)=\omega_{1} \cdot \mathcal{C}_{s}\left(e_{i j}\right)+\omega_{2} \cdot \mathcal{C}_{a}\left(e_{i j}\right)
$$


where $\mathcal{C}_{s}$ and $\mathcal{C}_{a}$ represent the shape cost and sampling cost. $\omega_{i}$ denotes the corresponding weight value. Based on our experiments, we assign them as $\omega_{1}=$ 0.8 and $\omega_{2}=0.2$. $\mathcal{C}_{s}$ and $\mathcal{C}_{a}$ are used to preserve the overall shape of the graph and prevent the generation of long edges. The definition of $\mathcal{C}_{s}$ is:

$$
\mathcal{C}_{s}\left(e_{i j}\right)=\mathcal{E}\left(\hat{v}, v_{i}\right)+\mathcal{E}\left(\hat{v}, v_{j}\right)
$$

where the error metrics $\mathcal{E}\left(\hat{v}, v_{i}\right)$ and $\mathcal{E}\left(\hat{v}, v_{j}\right)$ are the sum of distances of the merged node center $\hat{v}$ to all of the adjacent edges of $v_{i}$ and $v_{j}$, respectively:

$$
\left\{\begin{array}{l}
\mathcal{E}\left(\hat{v}, v_{i}\right)=\boldsymbol{X}^{T}\left(\sum_{<v_{i}, v_{k}>\in E}\left[\boldsymbol{a}_{\times} \mid \boldsymbol{b}\right]^{T}\left[\boldsymbol{a}_{\times} \mid \boldsymbol{b}\right]\right) \boldsymbol{X} \\
\boldsymbol{a}=\left(v_{i}-v_{k}\right) /\left\|v_{i}-v_{k}\right\| \text { and } \boldsymbol{b}=\boldsymbol{a} \times v_{i}
\end{array}\right.
$$

where $\boldsymbol{X}$ is a $4 \times 1$ vector that represents the homogeneous coordinate of the merging point. The sum operator represents the error metric $\mathcal{E}$ factorizing all the neighboring nodes $v_{k}$ that connects to $v_{i} . \boldsymbol{a}_{\times}$is the skew-symmetric matrix of the vector $\boldsymbol{a}$. Similarly, the definition of $\mathcal{E}\left(\hat{v}, v_{j}\right)$ can be derived by replacing the index $i$ by $j$ in Eq. (8). The basic idea behind these equations of $\mathcal{E}\left(\hat{v}, v_{i}\right)$ and $\mathcal{E}\left(\hat{v}, v_{j}\right)$ is to determine whether the nodes $v_{i}$ and $v_{j}$ have similar influences or contributions in forming the object shape based on their 3D positions. The influence of $v_{i}$ or $v_{j}$ is measured by how far its 3D position is deviated from its neighborhood on the mesh.

For the sampling cost term for $\mathcal{C}_{s}\left(e_{i j}\right)$, it is defined as:

$$
\mathcal{C}_{a}\left(e_{i j}\right)=\left\|v_{i}-v_{j}\right\| \sum_{<v_{i}, v_{k}>\in E}\left\|v_{i}-v_{k}\right\|
$$

The sampling cost is estimated based on two factors: the distance between $v_{i}$ and $v_{j}$; the sum of distances between $v_{i}$ and all its neighbors. The higher values of these distances, the lower chance of merging the two nodes $v_{i}$ and $v_{j}$. So in such a way, for each iteration, the edge with the lowest edge score is identified and the corresponding nodes are merged. This iterative process is repeated until there are no more collapsible node pairs can be merged according to the criteria in Eq. (5).

\subsection{Skin Attachment}

After nodes merging, a desirable embedded skeleton is constructed. Now we need to specify how each polygon transforms against the skeleton's deformation. Here we use the classic approach: linear blend skinning (LBS) that returns a new position for a $3 \mathrm{D}$ vertex based on its response weight to a particular skeleton transformation. To assign the weights between the skeleton bones and vertices, we use the heat equilibrium based approach by treating the character volume as an insulated heat-conducting body [33]. The analogy is drawn based on the observations: (1) To avoid folding artifacts, the distance between an effected surface point and its corresponding joint should be proportional to the width 
of a transition between two bones meeting at the joint. (2) To ensure smooth transition and natural deformation, gradient weight assignment is preferred over the vertices along surface.

\section{$6 \quad$ Experiment Results}

We evaluate the performance of our approach from two perspectives: pose estimation from a monocular video and skeleton extraction for given mesh. We have implemented the described approach in native $\mathrm{C}++$ with OpenGL, OpenCV libraries. The test is conducted on an Intel(R) Xeon(R)CPU E5-1620
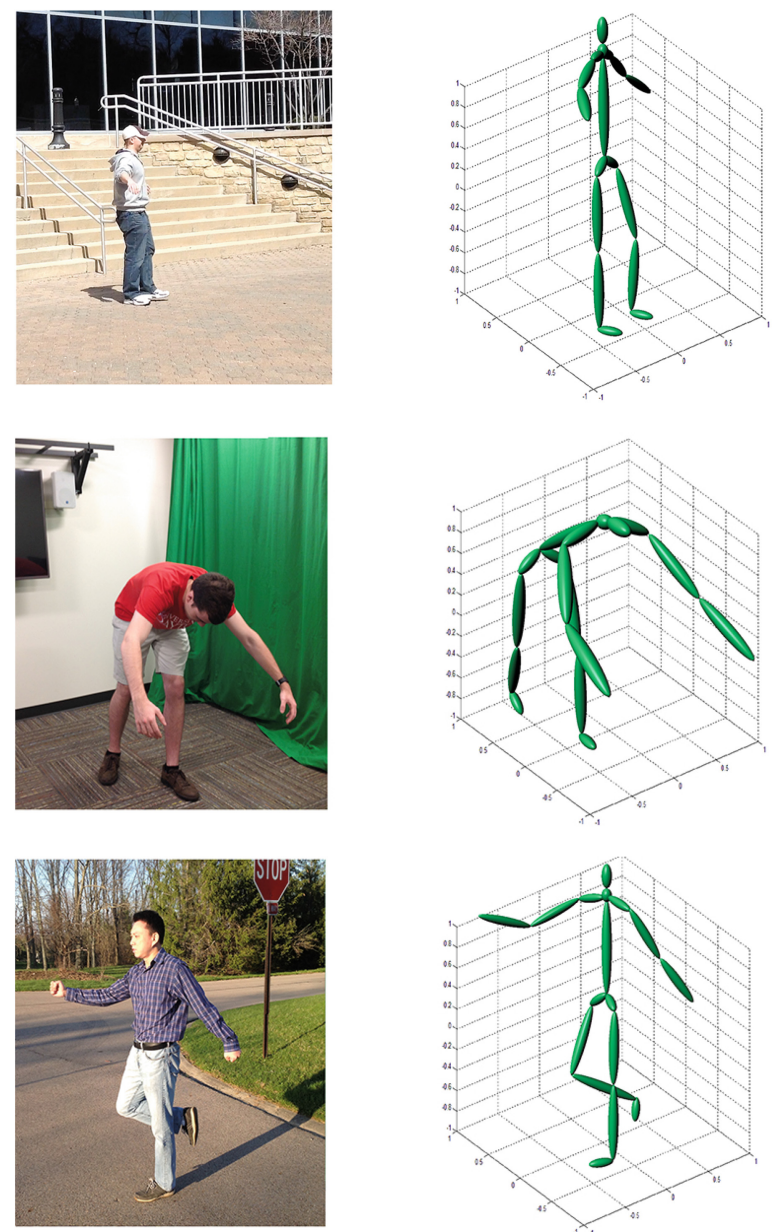

(a)
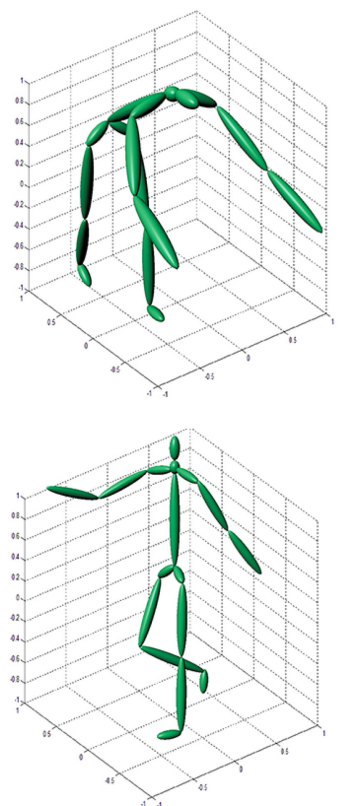

(b)
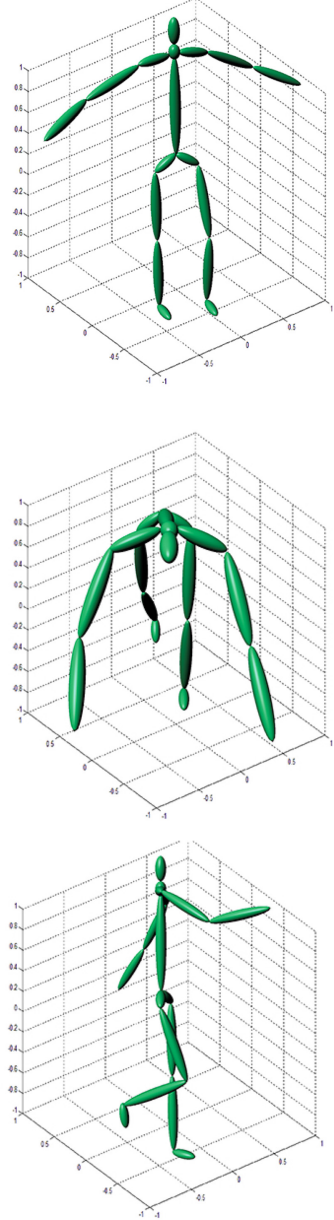

(c)

Fig. 4. Pose estimation results: (a) Input frames (b) Extracted 3D poses (c) An alternative view of the estimated poses 

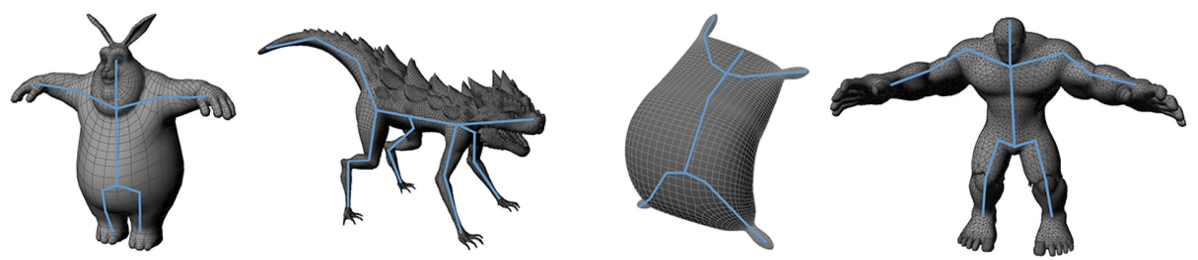

Fig. 5. Automatic skeletons extraction for various articulated models
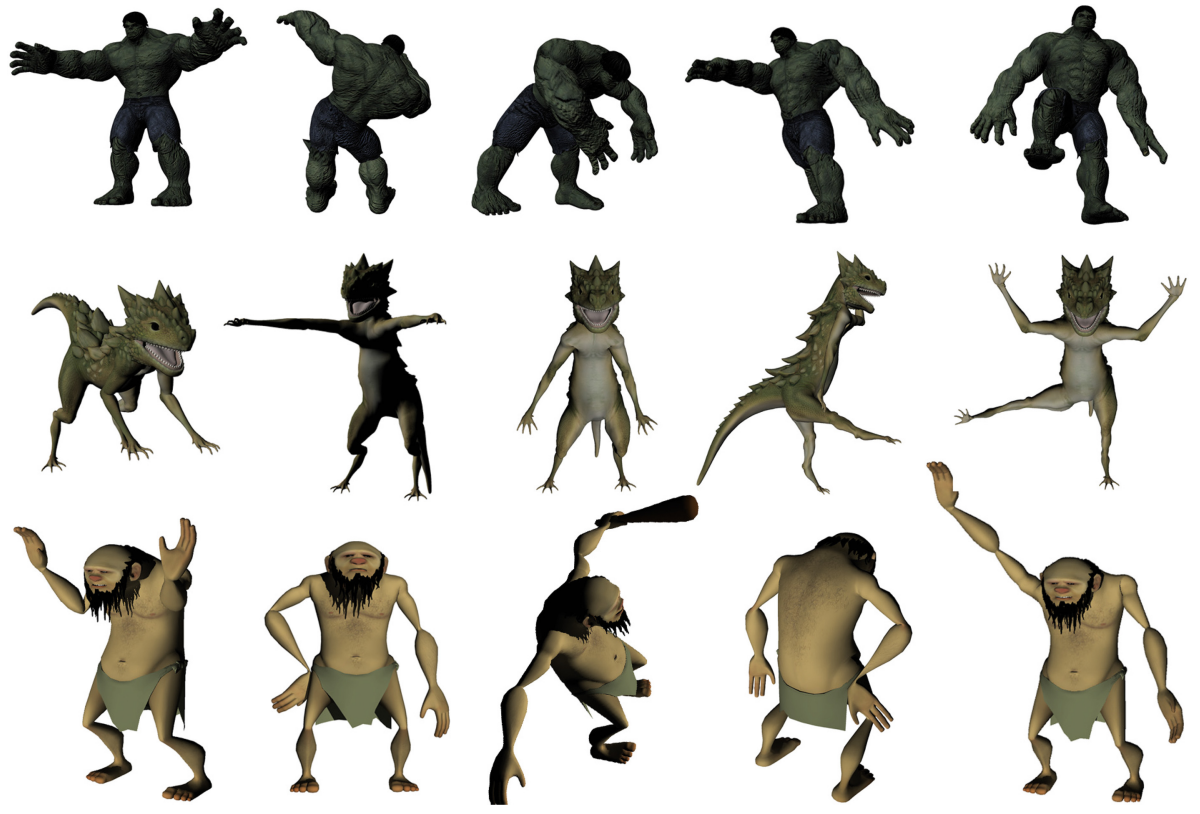

Fig. 6. Demonstration results animated by human pose from video

v3 $(3.5 \mathrm{GHz})$ with $24 \mathrm{~GB}$ RAM and NVIDIA GeForce GTX 980, with an an ordinary RGB camera producing $640 \times 480$ video frames at a frame rate of $30 \mathrm{fps}$. In our experiment, 5 users with different body movements are recorded by the video camera. The $3 \mathrm{D}$ mesh for the target characters are obtained directly from the public dataset ${ }^{1}$.

For the pose estimation evaluation, we tested it in different scenarios: from indoor environment to the outside; from front view with all the limbs presented in the video frame to side views with a certain amount of self-occlusion. The results of the estimated poses are demonstrated in Fig. 4. We select three frames from the captured video footages. As the ground truth is not available, a qualitative visual demonstration with different perspective views are presented. In Fig. 4, the first column shows the original input frame; the second and third column

\footnotetext{
${ }^{1}$ http://www.creativecrash.com/
} 
show the results of our 3D pose estimation. It is visually evident our approach can effectively handle the self-occlusion processing by processing multiple frames simultaneously to reduce the ambiguity.

Figure 5 shows the extracted kinematic skeletons and the deformation results after transferring the 3D pose from the monocular video demonstrated in Fig. 6. According to the mesh shapes, the potential graph is estimated by decomposing the whole surface into multiple components, based on which the potential skeleton can be further inferred. Our method can successfully extract the embedded skeleton for those characters with similar structures as human body. However, for other special shape objects, even users can manually select skeleton correspondences, the generated animation may not look realistic due to the different topology structures. This will be our next step of research for further improvement.

\section{Conclusion}

We have presented a new way for automatic animation given an arbitrary 3D character. Human motion captured from a single video camera is used as input and transferred to the target mesh. The goal of our framework is to provide novice users with the flexibility and simplicity to create animations on a 3D character. By automatically extracting the skeletons from the target object and extracting 3D poses from the video, the animation process can be potentially simplified. Our results are preliminary but promising. In the future, in order to provide more visually realistic animations, we would like to do further research with robust geometrical analysis for more special shaped objects, especially for those with distinct structures as human body.

\section{References}

1. Igarashi, T., Matsuoka, T., Hughes, J.F.: Spatial keyframing for performancedriven animation. In: Symposium on Computer Animation (SCA) (2005)

2. Thorne, M., Burke, D., Van, M.: Motion doodles: an interface for sketching character motion. ACM Trans. Graph. 23(3), 424-431 (2004)

3. Shen, J., Cheung, S.: Layer depth denoising and completion for structured-light RGB-D cameras. In: IEEE Conference on Computer Vision and Pattern Recognition (CVPR), pp. 1187-1194 (2013)

4. Shen, J., Su, P.-C., Cheung, S.-C., Zhao, J.: Virtual mirror rendering with stationary RGB-D cameras and stored 3-D background. IEEE Trans. Image Process. 22, 3433-3448 (2013)

5. Bloomenthal, J., Lim, C.: Skeletal methods of shape manipulation. In: Shape Modeling International Conference (1999)

6. Katz, S., Tal, A.: Hierarchical mesh decomposition using fuzzy clustering and cuts. In: ACM SIGGRAPH (2003)

7. Gagvani, N., Silver, D.: Animating volumetric models. In: Graphical Models (2001)

8. Lien, J., Keyser, J., Amato, N.: Simultaneous shape decomposition and skeletonization. In: ACM SPM/SMI (2006)

9. Shen, J., Yang, J., Taha-abusneineh, S., Payne, B., Hitz, M.: Structure preservinglarge imagery reconstruction. J. Cyber Secur. Mobil. 3(3) (2014) 
10. Yang, J., Wang, Y., Wang, H., Hua, K., Wang, W., Shen, J.: Automatic objects removal for scene completion. In: Workshop on Security and Privacy in Big Data, The 33rd Annual IEEE International Conference on Computer Communications (INFOCOM 2014), Toronto, Canada (2014)

11. Zhou, Y., Li, L., Zhao, T., Zhang, H.: Region-based high-level semantics extraction with CEDD. In: IEEE International Conference on Network Infrastructure and Digital Content (2010)

12. Wang, J., Halima, B., Gao, X.: Joint learning and weighting of visual vocabulary for bag-of-feature based tissue classification. Pattern Recogn. 46, 3249-3255 (2013)

13. Shen, J., Tan, W.: Image-based indoor place-finder using image to plane matching. In: IEEE International Conference on Multimedia and Expo (ICME) (2014)

14. Zhou, Y., Li, Le, Zhao, T., Zhang, H.: Adaptive Learning of Region-based pLSA Model for Total Scene Annotation (2013). arXiv preprint arXiv:1311.5590

15. Li, L., Yang, J., Zhao, K., Xu, Y., Zhang, H., Fan, Z.: Graph Regularized Nonnegative Matrix Factorization By Maximizing Correntropy (2014). arXiv preprint arXiv:1405.2246

16. Shotton, J., Fitzgibbon, A., Cook, M., Sharp, T., Finocchio, M.: Real-time human pose recognition in parts from a single depth image. In: IEEE CVPR (2011)

17. Bregler, C., Hertzmann, A., Biermann, H.: Recovering non-rigid 3D shape from image streams. In: CVPR (2000)

18. Yang, Y., Ramanan, D.: Articulated pose estimation with flexible mixtures-ofparts. In: IEEE CVPR (2011)

19. Wang, Y., Nandi, A., Agrawal, G.: SAGA: array storage as a DB with support for structural aggregations. In: Proceedings of SSDBM (2014)

20. Ma, J., Choi, S.: Kinematic skeleton extraction from 3D articulated models. Comput. Aided Des. 46, 221-226 (2014)

21. Leordeanu, M., Hebert, M.: A spectral technique for correspondence problems using pairwise constraints. In: IEEE International Conference on Computer Vision (2005)

22. Garland, M., Heckbert, S.: Surface simplification using quadric error metrics. In: Proceedings of SIGGRAPH (1997)

23. Sumner, R.W., Schmid, J., Pauly, M.: Embedded deformation for shape manipulation. In: ACM SIGGRAPH (2007)

24. Luo, J., Alexander B.: A Heaviside-based regression of piecewise functions expressed as object-oriented programs. In: The International Conference on Machine Learning and Computing (2011)

25. Alexander, B., Luo, J., Nash, H.: CoReJava: learning functions expressed as Object-Oriented programs. In: IEEE International Conference on In Machine Learning and Applications (2008)

26. Wang, J., Bensmail, H., Yao, N., Gao, X.: Discriminative sparse coding on multimanifolds. Knowl. Based Syst. 54, 199-206 (2013)

27. Yang, Y., Ramanan, D.: Articulated pose estimation with flexible mixtures-ofparts. In: IEEE CVPR 2011 (2011)

28. Wang, Y., Agrawal, G., Ozer, G., Huang, K.: Removing sequential bottlenecks in analysis of next-generation sequencing data. In: Workshop on High Performance Computational Biology (HiCOMB), IEEE International Parallel Distributed Processing Symposium (2014)

29. Ramanan, D.: Learning to parse images of articulated bodies. In: NIPS (2006)

30. Ferrari, V., Marn-Jimnez, M., Zisserman, A.: 2D human pose estimation in TV shows. In: Proceedings of the Dagstuhl Seminar on Statistical and Geometrical Approaches to Visual Motion Analysis (2009) 
31. Sigal, L., Black, M.J.: Measure locally, reason globally: occlusion-sensitive articulated pose estimation. In: CVPR (2006)

32. Quan, L., Lan, Z.: Linear N-point camera pose determination. IEEE Tras. PAMI 21, 774-780 (1999)

33. Baran, I., Popovic, J.: Automatic rigging and animation of $3 \mathrm{D}$ characters. In: SIGGRAPH (2007)

34. Wang, J., Bensmail, H., Gao, X.: Multiple graph regularized protein domain ranking. BMC Bioinf. 13, 307 (2012) 